\title{
Assouad's theorem with dimension independent of the snowflaking
}

\author{
Assaf Naor and Ofer Neiman
}

\begin{abstract}
It is shown that for every $K>0$ and $\varepsilon \in(0,1 / 2)$ there exist $N=N(K) \in \mathbb{N}$ and $D=D(K, \varepsilon) \in(1, \infty)$ with the following properties. For every metric space $(X, d)$ with doubling constant at most $K$, the metric space $\left(X, d^{1-\varepsilon}\right)$ admits a bi-Lipschitz embedding into $\mathbb{R}^{N}$ with distortion at most $D$. The classical Assouad embedding theorem makes the same assertion, but with $N \rightarrow \infty$ as $\varepsilon \rightarrow 0$.
\end{abstract}

\section{Introduction}

In this paper all metric spaces are assumed to be separable and to contain at least two points. Balls in metric spaces are always closed balls, i.e., for a metric space $(X, d), x \in X$ and $r \geqslant 0$, we denote $B(x, r)=\{y \in X: d(x, y) \leqslant r\}$. A metric space $(X, d)$ has doubling constant $K \in(1, \infty)$ if every ball in $X$ can be covered by at most $K$ balls of half its radius, i.e., for every $x \in X$ and $r>0$ there exist $A \subseteq X$ with $|A| \leqslant K$ such that $B(x, r) \subseteq \bigcup_{y \in A} B(y, r / 2)$. Note that since $X$ contains at least two points, necessarily $K \geqslant 2$. $(X, d)$ is said to be a doubling metric space if it has doubling constant $K$ for some $K \in(1, \infty)$.

A metric space $(X, d)$ embeds into a normed space $(Y,\|\cdot\|)$ with distortion $D \in[1, \infty]$ if there exists $f: X \rightarrow Y$ such that for all $x, y \in X$ we have

$$
d(x, y) \leqslant\|f(x)-f(y)\| \leqslant D d(x, y) .
$$

When $X$ embeds into $Y$ with finite distortion we say that $X$ admits a bi-Lipschitz embedding into $Y$. The infimum over those $D \geqslant 1$ for which $X$ embeds into $Y$ is denoted $c_{Y}(X)$. When $Y=\ell_{2}$ is infinite dimensional Hilbert space, we write $c_{Y}(X)=c_{2}(X)$; this parameter is known in the literature as the Euclidean distortion of $X$. In what follows, when we refer to the space $\mathbb{R}^{N}$ we always assume that it is equipped with the standard Euclidean metric. A standard argument

Mathematics Subject Classification (2010): Primary 46B85; Secondary 51F99.

Keywords: Doubling metric spaces, Assouad's theorem. 
(see, e.g., Lemma 4.9 in [4]) shows that if $Y$ is either $\ell_{2}$ or $\mathbb{R}^{N}$, we have

$$
c_{Y}(X)=\sup \left\{c_{Y}(Z): Z \subseteq X \wedge|Z|<\infty\right\} .
$$

If $(X, d)$ is a metric space and $\alpha \in(0,1]$ then $\left(X, d^{\alpha}\right)$ is also a metric space, known as the $\alpha$-snowflake of $X$.

A major open problem of embedding theory is the bi-Lipschitz embeddability problem in $\mathbb{R}^{N}$. This problem asks for an intrinsic characterization of those separable metric spaces $(X, d)$ that admit a bi-Lipschitz embedding into $\mathbb{R}^{N}$ for some $N \in \mathbb{N}$. For a discussion of this important question, see for example the works of Semmes [28], Lang-Plaut [20] and Heinonen [16]. An obvious restriction on a metric space $(X, d)$ that admits a bi-Lipschitz embedding into $\mathbb{R}^{N}$ is that it must be doubling. In this context, Assouad discovered in [4] the following fundamental embedding theorem (see also Heinonen's book [15] for a nice exposition of Assouad's theorem).

Theorem 1.1 (Assouad's embedding theorem). For every $\varepsilon \in(0,1)$ and $K>0$, there exist $N=N(K, \varepsilon) \in \mathbb{N}$ and $D=D(K, \varepsilon) \in(1, \infty)$ such that for every separable metric space $(X, d)$ with doubling constant $K$, the metric space $\left(X, d^{1-\varepsilon}\right)$ admits a bi-Lipschitz embedding into $\mathbb{R}^{N}$ with distortion at most $D$.

Assouad's theorem falls short of solving the bi-Lipschitz embeddability problem in $\mathbb{R}^{N}$, since it only achieves an embedding of the snowflaked metric space $\left(X, d^{1-\varepsilon}\right)$. Nevertheless, as $\varepsilon \rightarrow 0$ this metric space becomes closer and closer to the original metric space $(X, d)$. It is therefore of interest to investigate the behavior of $N(K, \varepsilon)$ and $D(K, \varepsilon)$ as $\varepsilon \rightarrow 0$. It turns out that necessarily $\lim _{\varepsilon \rightarrow 0} D(K, \varepsilon)=\infty$, due to the existence of doubling metric spaces that do not admit a bi-Lipschitz embedding into $\mathbb{R}^{N}$. The first known such example is the Heisenberg group, equipped with the Carnot-Carathéodory metric: Semmes observed in [27] that its bi-Lipschitz nonembeddability into $\mathbb{R}^{N}$ is a consequence of Pansu's differentiability theorem [25]. Additional examples of non-Euclidean doubling spaces were found by Laakso [19] and Bourdon-Pajot [7]; see the work of Cheeger [8] for a unified treatment of these results.

It seems to be inherent to Assouad's embedding method that we also have $\lim _{\varepsilon \rightarrow 0} N(K, \varepsilon)=\infty$. Note that if $\varepsilon \in(0,1 / 2)$ then the metric space $\left(X, d^{1-\varepsilon}\right)$ has doubling constant $K^{2}$, so there is no obvious obstruction to $\left(X, d^{1-\varepsilon}\right)$ admitting a bi-Lipschitz embedding into $\mathbb{R}^{N}$ for some $N$ that is independent of $\varepsilon \in(0,1 / 2)$. The issue that in Assouad's theorem $N$ depends on $\varepsilon$ and is very large as $\varepsilon \rightarrow 0$ was noted by many authors; this is mentioned, for example, in the works of DavidToro [10] and Semmes [28] (where much more refined bounds on $N$ are obtained under additional assumptions). Assouad himself noticed this issue in [4], where he showed that $N$ can be taken to be independent of $\varepsilon \in(0,1 / 2)$ when $X=\mathbb{R}$ (more generally, Assouad deals in [4] with $X=[0,1]^{k}$ ). The case of the "helix snowflakes" ( $\left.\mathbb{R},|x-y|^{1-\varepsilon}\right)$ was studied by Kahane [17] and Talagrand [29], who investigated the interplay between the dimension $N$ and the distortion $D$ (Kahane studied only the case $\varepsilon=\frac{1}{2}$, and obtained sharp results. Talagrand's work applies to all $\varepsilon \in(0,1)$, but is not sharp). 
Here we show that in Assouad's theorem one can take $N$ to depend only on the doubling constant $K$, but not on $\varepsilon \in(0,1 / 2)$.

Theorem 1.2. For every $K>0$ there exists $N=N(K) \in \mathbb{N}$, and for every $\varepsilon \in(0,1 / 2)$ and $K>0$ there exists $D=D(K, \varepsilon) \in(1, \infty)$, such that for every separable metric space $(X, d)$ with doubling constant $K$, the metric space $\left(X, d^{1-\varepsilon}\right)$ admits a bi-Lipschitz embedding into $\mathbb{R}^{N}$ with distortion at most $D$.

Our argument yields the bounds $N(K) \lesssim \log K$ and $D(K, \varepsilon) \lesssim\left(\frac{\log K}{\varepsilon}\right)^{2}$. More generally, for every $\delta \in(0,1]$ our argument yields the bounds

$$
N(K) \lesssim \frac{\log K}{\delta} \text { and } D(K, \varepsilon) \lesssim\left(\frac{\log K}{\varepsilon}\right)^{1+\delta} .
$$

Here and in what follows, the symbols $\lesssim, \gtrsim$ indicate the corresponding inequalities up to an absolute multiplicative factor.

In the rest of this introduction we will describe some additional results and question related to the bi-Lipschitz embeddability problem in $\mathbb{R}^{N}$.

\subsection{The Lang-Plaut problem and snowflakes of the Heisenberg group}

Despite major efforts by many mathematicians, the bi-Lipschitz embeddability problem in $\mathbb{R}^{N}$ remains wide open. A variety of sufficient intrinsic conditions on a metric space $(X, d)$ are known which ensure that it admits a bi-Lipschitz embedding in some Euclidean space $\mathbb{R}^{N}$, but these conditions are far from necessary.

A necessary condition for a metric space $(X, d)$ to admit a bi-Lipschitz embedding into some $\mathbb{R}^{N}$ (in addition to being doubling) is that it admits a bi-Lipschitz embedding into $\ell_{2}$, i.e., its Euclidean distortion satisfies $c_{2}(X)<\infty$. All the known examples of doubling metric spaces that do not admit a bi-Lipschitz embedding into any $\mathbb{R}^{N}$ actually do not admit a bi-Lipschitz embedding into infinite dimensional Hilbert space as well. This led Lang and Plaut (see Question 2.4 in [20]) to ask the following question:

Question 1 (Lang-Plaut problem). Is it necessary and sufficient for a metric space $(X, d)$ to admit a bi-Lipschitz embedding into some $\mathbb{R}^{N}$ that it is doubling and it admits a bi-Lipschitz embedding into Hilbert space? Equivalently, does every doubling subset of Hilbert space admit a bi-Lipschitz embedding into some $\mathbb{R}^{N}$ ?

By a simple argument (presented in Section 4), the Lang-Plaut problem can be restated quantitatively as follows: Is it true that for every $K>0$ there is $N=N(K) \in \mathbb{N}$ and $D=D(K) \in(1, \infty)$ such that if $X \subseteq \ell_{2}$ has doubling constant $K$ then $c_{\mathbb{R}^{N}}(X) \leqslant D$ ?

One might argue whether or not a positive answer to the Lang-Plaut problem would resolve the bi-Lipschitz embedding problem into $\mathbb{R}^{N}$, since it is not obvious that the condition that $X$ admits a bi-Lipschitz embedding into Hilbert space can be restated in terms of the intrinsic geometry of $X$. However, it is possible to characterize bi-Lipschitz embeddability into $\ell_{2}$ in terms of a family of distance inequalities, i.e., intrinsically, without using the word "embedding". Indeed, as shown by Linial, London and Rabinovich [23] (extending the corresponding classical result 
of Schoenberg [26] in the isometric category), $c_{2}(X) \leqslant D$ if and only if for all $n \in \mathbb{N}, x_{1}, \ldots, x_{n} \in X$ and every $n \times n$ symmetric positive semidefinite matrix $Q=\left(q_{i j}\right)$, all of whose rows sum to 0 , the following inequality holds true:

$$
\sum_{i=1}^{n} \sum_{j=1}^{n} \max \left\{q_{i j}, 0\right\} d\left(x_{i}, x_{j}\right)^{2} \leqslant D^{2} \sum_{i=1}^{n} \sum_{j=1}^{n} \max \left\{-q_{i j}, 0\right\} d\left(x_{i}, x_{j}\right)^{2} .
$$

Hence, a positive answer to the Lang-Plaut question would yield a characterization of bi-Lipschitz embeddability into some $\mathbb{R}^{N}$ in terms of the doubling condition, and the family of distance inequalities (1.3). We believe that this would yield a satisfactory answer to the bi-Lipschitz embeddability problem in $\mathbb{R}^{N}$, though there does not seem to be evidence supporting a positive answer to the the Lang-Plaut question.

A potential source of doubling subsets of Hilbert space that might yield a counterexample to the Lang-Plaut problem is Assouad's theorem itself. When allowing embeddings into infinite dimensional Hilbert space rather than into $\mathbb{R}^{N}$, the asymptotics in terms of $\varepsilon$ of $D(K, \varepsilon)$ in Assouad's theorem are known [21] (see also [24]). Specifically, if $(X, d)$ has doubling constant $K$ then $c_{2}(X) \leqslant C(K) / \sqrt{\varepsilon}$ for some $C(K) \in(0, \infty)$. This dependence on $\varepsilon$ is sharp up to the value of $C(K)$, as shown in Remark 5.4 of [21].

If $(X, d)$ has doubling constant $K$ then the space $\left(X, d^{1-\varepsilon}\right)$ has doubling constant bounded uniformly in $\varepsilon \in(0,1 / 2)$, but, in its $C(K) / \sqrt{\varepsilon}$-distortion embedding into $\ell_{2}$ it might have an image that is not a doubling subset of $\ell_{2}$, with doubling constant independent of $\varepsilon$, due to the large distortion. We therefore ask the following question:

Question 2. Is it true that for every $K \in(1, \infty)$ there exist $a(K), b(K) \in(0, \infty)$ with the following property. If $(X, d)$ has doubling constant $K$ and $\varepsilon \in(0,1 / 2)$ then there exists $f: X \rightarrow \ell_{2}$ such that $a(K) \sqrt{\varepsilon} d(x, y)^{1-\varepsilon} \leqslant\|f(x)-f(y)\| \leqslant d(x, y)^{1-\varepsilon}$ for all $x, y \in X$, and $f(X) \subseteq \ell_{2}$ has doubling constant $b(K)$.

Observe that due to Theorem 1.2, with the explicit bounds stated in (1.2), if we replaced in Question 2 the term $\sqrt{\varepsilon}$ by $\varepsilon^{1+\delta}$ for any $\delta \in(0,1]$, then the answer would be positive, and even the image of the embedding would be finite dimensional with dimension depending only on $K$ and $\delta$.

In spite of the fact that we don't know the answer to Question 2, we do know that the answer is positive for the Heisenberg group. For $n \in \mathbb{N}$, the $n$th Heisenberg group $\mathbb{H}_{n}$ is $\mathbb{C}^{n} \times \mathbb{R}$, equipped with the following group product:

$$
\begin{aligned}
& (w, s) \cdot(z, t)=\left(w+z, s+t+2 \sum_{j=1}^{n} \Im\left(w_{j} \overline{z_{j}}\right)\right) \\
& \forall w=\left(w_{1}, \ldots, w_{n}\right), z=\left(z_{1}, \ldots, z_{n}\right) \in \mathbb{C}^{n}, \forall s, t \in \mathbb{R} .
\end{aligned}
$$

Thus $(0,0)$ is the identity of $\mathbb{H}_{n}$ and for $(z, t) \in \mathbb{H}_{n}$ we have $(z, t)^{-1}=(-z,-t)$.

The Koranyi norm on $\mathbb{H}_{n}$ is defined for $(z, t) \in \mathbb{H}_{n}$ by $N_{0}(z, t)=\sqrt[4]{|z|^{4}+t^{2}}$, where $|z|^{2}=\sum_{j=1}^{n}\left|z_{j}\right|^{2}$. For $g, h \in \mathbb{H}_{n}$ we have $N_{0}\left(g h^{-1}\right) \leqslant N_{0}(g)+N_{0}(h)$ 
(see [18], [9]). Thus $d_{N_{0}}(g, h)=N_{0}\left(h^{-1} g\right)$ is a left-invariant metric on $\mathbb{H}_{n}$. One can check that the Lebesgue measure is a Haar measure of $\mathbb{H}_{n}$, and that $\left(\mathbb{H}_{n}, d_{N_{0}}\right)$ has doubling constant $e^{O(n)}$.

In Section 4 we observe that a result of [22] implies the following statement:

Theorem 1.3. For every $\varepsilon \in(0,1 / 2), n \in \mathbb{N}$, there exists $f_{\varepsilon}: \mathbb{H}_{n} \rightarrow \ell_{2}$ satisfying $\sqrt{\varepsilon} d_{N_{0}}(x, y)^{1-\varepsilon} \leqslant\left\|f_{\varepsilon}(x)-f_{\varepsilon}(y)\right\| \leqslant d_{N_{0}}(x, y)^{1-\varepsilon}$ for all $x, y \in \mathbb{H}_{n}$, and such that $f_{\varepsilon}\left(\mathbb{H}_{n}\right)$ is a doubling subset of $\ell_{2}$, with doubling constant $e^{O(n)}$.

We also show in Section 4 that Theorem 1.3 is sharp, even without the requirement that the image of $\mathbb{H}_{n}$ be doubling with constant independent of $\varepsilon$ :

$$
c_{2}\left(\mathbb{H}_{n}, d_{N_{0}}^{1-\varepsilon}\right) \gtrsim \frac{1}{\sqrt{\varepsilon}} \quad \forall \varepsilon \in(0,1 / 2) .
$$

This raises the following question:

Question 3. Is it true that for every fixed $N \in \mathbb{N}$ we have

$$
\lim _{\varepsilon \rightarrow 0} c_{\mathbb{R}^{N}}\left(\mathbb{H}_{1}, d_{N_{0}}^{1-\varepsilon}\right) \sqrt{\varepsilon}=\infty ?
$$

A positive answer to Question 3 would imply a negative answer to the LangPlaut problem, since otherwise there would be $N \in \mathbb{N}$ and $D \in(1, \infty)$ satisfying $c_{\mathbb{R}^{N}}\left(f_{\varepsilon}\left(\mathbb{H}_{1}\right)\right) \leqslant D$ for all $\varepsilon \in(0,1 / 2)$, where $f_{\varepsilon}$ is the Euclidean embedding of $\left(\mathbb{H}_{1}, d_{N_{0}}^{1-\varepsilon}\right)$ from Theorem 1.3. This would yield the bound $c_{\mathbb{R}^{N}}\left(\mathbb{H}_{1}, d_{N_{0}}^{1-\varepsilon}\right) \sqrt{\varepsilon} \leqslant D$.

\subsection{Previous work and an overview of the proof of Theorem $\mathbf{1 . 2}$}

The classical proof of Assouad's theorem [4] (see also [15]) yields the dimension bound $N(K, \varepsilon) \leqslant c(K) / \varepsilon^{O(1)}$. In [13], Gupta, Krauthgamer and Lee announced a similar bound on $N(K, \varepsilon)$ with a much better dependence of $c(K)$ on $K$, yet the same bound in terms of $\varepsilon$ (the proof of this assertion of [13] has not appeared since the 2003 announcement, and in particular the dependence on $\varepsilon$ was not stated there explicitly, but it seems to us that the proof technique suggested in [13] would lead to this bound). A similar bound follows from the work of Har-Peled and Mendel [14], who studied in addition embeddings into $\ell_{\infty}^{N}$, yielding a $1+\delta$ distortion result. The best previously known bound is due to Abraham, Bartal and Neiman [1], who proved that $N(K, \varepsilon) \leqslant c(K) \log (1 / \varepsilon)$. In the context of the Lang-Plaut problem, Gottlieb and Krauthgamer [12], and Bartal, Recht and Schulman [6], proved that if $X \subseteq \ell_{2}$ has doubling constant $K$ then for all $\delta \in(0,1)$ the $(1-\varepsilon)$-snowflake of $X$ embeds with distortion $1+\delta$ into $\mathbb{R}^{c(K, \delta) / \varepsilon^{O(1)}}$; the main point in these works, however, is to obtain a $1+\delta$ distortion embedding, which is impossible in the context of general doubling metric spaces that are not necessarily isometric to a subset of $\ell_{2}$.

Our proof of Theorem 1.2 builds heavily on the method of Abraham-BartalNeiman [1]. In essence, our proof should be viewed as an optimization of the argument of [1], which uses degrees of freedom that were available in the construction of [1], but has not been previously exploited. This requires subtle changes in 
in the proof of [1], and in particular we were surprised that such changes can lead to a complete removal of the dependence on $\varepsilon$ of the dimension $N$ in Assouad's theorem. Though somewhat delicate, these changes are of a technical nature, and the key conceptual ideas can be found in [1].

The proof of Theorem 1.2 is based on a construction of a distribution over random embeddings, arising from a certain family of random multi-scale partitions of the metric space $(X, d)$. At every possible distance scale we provide a mapping to $\mathbb{R}$ which is essentially the truncated distance to the "boundary" of the random partition. We then combine all the possible scales into a single embedding into $\mathbb{R}$, using an idea of Assouad [4] which multiplies every scale by an appropriate factor that enables us to control the total expansion over all scales. The lower bound on the distance of the image of every pair of points in $X$ will come from a single critical scale. Instead of showing sufficient contribution for every pair, we first focus on certain nets of the space at appropriate scales, showing that this suffices to prove the desired lower bound on all pairs. The bulk of the proof consists of arguing that not only the net pairs will have sufficient contribution, but that this will happen with high probability (depending on $\varepsilon$ ), and with very few dependencies on other net points. To show this we use, as in [1], a localization property of the "padding event" of the random partitions: this event is stochastically independent of the "far away" structure of the partition. The ball expected to be padded is very small (which causes additional distortion), but on the other hand the padding probability is high. The fact that the radius of our padded ball is small (as a function of $\varepsilon$ ) forces us to define the original distance scales to be also functions of $\varepsilon$. Finally, to argue that the desired lower bound happens for all pairs with positive probability, even though the number of dimensions at our disposal is small, we use the Lovász Local Lemma.

Acknowledgements. We are grateful to Tim Austin and Bruce Kleiner for helpful discussions.

\section{Preliminaries}

Due to (1.1), it suffices to prove Theorem 1.2 when $X$ is finite, provided that the resulting distortion $D(K, \varepsilon)$ and the dimension $N(K)$ do not depend on $|X|$. We will therefore assume from now on that $X$ is finite. This assumption is actually not necessary for our argument, but it serves the role of allowing us to ignore measurability issues that might arise in the random partitioning arguments.

For a partition $P$ of $X$ and $x \in X$ let $P(x) \in P$ be the set in $P$ to which $x$ belongs. For $s>0$ the partition $P$ is called $s$-bounded if the diameter of $P(x)$ is at most $s$ for all $x \in X$.

There is a canonical way to obtain partitions from balls. Given $x_{1}, \ldots, x_{n} \in X$ and $r_{1}, \ldots, r_{n} \in(0, \infty)$, define a partition $P_{r_{1}, \ldots, r_{n}}^{x_{1}, \ldots, x_{n}}$ of $\bigcup_{j=1}^{n} B\left(x_{j}, r_{j}\right)$ by

$$
P_{r_{1}, \ldots, r_{n}}^{x_{1}, \ldots, x_{n}} \stackrel{\text { def }}{=}\left\{B\left(x_{1}, r_{1}\right)\right\} \bigcup\left\{B\left(x_{j}, r_{j}\right) \backslash \bigcup_{i=1}^{j-1} B\left(x_{i}, r_{i}\right)\right\}_{j=2}^{n} \backslash\{\emptyset\} .
$$


In particular, given $s>0$ the partition $P_{r_{1}, \ldots, r_{n}}^{x_{1}, \ldots, x_{n}}$ is an $s$-bounded partition of $X$ whenever $\left\{x_{1}, \ldots, x_{n}\right\}$ is an $s / 4$-net of $X$ and $r_{1}, \ldots, r_{n} \in[s / 4, s / 2]$.

As in [1], we will use random partitions of the form $P_{r_{1}, \ldots, r_{n}}^{x_{1}, \ldots, x_{n}}$, where the radii $r_{1}, \ldots, r_{n}$ are appropriately chosen random variables. We present the proofs of the necessary properties of these partitions below, even though they follow from [1]. We do so since the argument of [1] is carried out in much greater generality because in [1] these methods are used for other purposes for which more general constructions are needed. Our argument below is simpler than the proof in [1] both because it deals with the special case that we need, but also because the proof here is different from [1] (relying, of course, on the same ideas).

Lemma 2.1. Fix $x \in X$. For $s>0$ and $K>1$, let $R$ be a random variable with the following density:

$$
\phi_{s}(r) \stackrel{\text { def }}{=} \frac{16 K^{8} \log K}{s\left(K^{4}-1\right)} K^{-16 r / s} \mathbf{1}_{[s / 4, s / 2]}(r) .
$$

Then for every $\beta>0$ and every $y \in X$ we have

$$
\begin{aligned}
\mathbb{P}[B(x, R) \cap B(y, \beta s) & \notin\{\emptyset, B(y, \beta s)\}] \\
& \leqslant\left(1-K^{-32 \beta}\right)\left(\mathbb{P}[B(y, \beta s) \cap B(x, R) \neq \emptyset]+\frac{1}{K^{4}-1}\right) .
\end{aligned}
$$

Proof. Fix $x, y \in X$ and define

$$
a \stackrel{\text { def }}{=} \min _{z \in B(y, \beta s)} d(x, z) \quad \text { and } \quad b \stackrel{\text { def }}{=} \max _{z \in B(y, \beta s)} d(x, z) .
$$

By the triangle inequality,

$$
b-a \leqslant 2 \beta s .
$$

Hence,

$$
\begin{aligned}
\mathbb{P}[B(x, R) \cap B(y, \beta s) & \notin\{\emptyset, B(y, \beta s)\}]=\int_{\max \{a, s / 4\}}^{b} \phi_{s}(r) d r \\
& \leqslant \frac{K^{8}}{K^{4}-1}\left(K^{-16 \max \{a, s / 4\} / s}-K^{-16 b / s}\right) \\
& \stackrel{(2.4)}{ } \leqslant \frac{K^{8}}{K^{4}-1} K^{-16 \max \{a, s / 4\} / s}\left(1-K^{-32 \beta}\right) .
\end{aligned}
$$

Similarly,

$$
\begin{aligned}
\mathbb{P}[B(y, \beta s) \cap B(x, R) \neq \emptyset] & =\int_{\max \{a, s / 4\}}^{s / 2} \phi_{s}(r) d r \\
& =\frac{K^{8}}{K^{4}-1}\left(K^{-16 \max \{a, s / 4\} / s}-K^{-8}\right) .
\end{aligned}
$$

The desired inequality (2.3) now follows from (2.5) and (2.6). 
Lemma 2.2. Fix $s>0, K \geqslant 2$. Assume that $(X, d)$ has doubling constant at most $K$. Let $\left\{x_{1}, \ldots, x_{n}\right\} \subseteq X$ be an $s / 4$-net of $X$, and let $R_{1}, \ldots, R_{n}$ be i.i.d. random variables whose distribution is given by (2.2). Then, for every $y \in X$ and $\beta \in(0,1 / 40)$, we have

$$
\mathbb{P}\left[B(y, \beta s) \subseteq P_{R_{1}, \ldots, R_{n}}^{x_{1}, \ldots, x_{n}}(y)\right] \geqslant K^{-64 \beta} .
$$

Proof. For every $j \in\{1, \ldots, n\}$ consider the following event:

$A_{j} \stackrel{\text { def }}{=}\left(\bigcap_{i=1}^{j-1}\left\{B\left(x_{i}, R_{i}\right) \cap B(y, \beta s)=\emptyset\right\}\right) \cap\left\{B\left(x_{j}, R_{j}\right) \cap B(y, \beta s) \notin\{\emptyset, B(y, \beta s)\}\right\}$.

For $A_{j}$ to occur we need in particular to have $B\left(x_{j}, R_{j}\right) \cap B(y, \beta s) \neq \emptyset$. Since $R_{j}, \beta s \leqslant s / 2$, this implies that $j \in J_{y}$, where

$$
J_{y} \stackrel{\text { def }}{=}\left\{j \in\{1, \ldots, n\}: x_{j} \in B(y, s)\right\} .
$$

We can cover $B(y, s)$ by at most $K^{3}$ balls of radius $s / 8$. Since $\left\{x_{1}, \ldots, x_{n}\right\}$ is an $s / 4$-net, each of these balls can contain at most one of the $x_{i}$. Thus,

$$
\left|J_{y}\right| \leqslant K^{3} \text {. }
$$

We must have $B\left(x_{j}, R_{j}\right) \cap B(y, \beta s) \neq \emptyset$ for some $j \in J_{y}$, and therefore using the independence of $R_{1}, \ldots R_{n}$ we see that

$$
\begin{aligned}
1 & =\sum_{j \in J_{y}} \mathbb{P}\left[B\left(x_{j}, R_{j}\right) \cap B(y, \beta s) \neq \emptyset \wedge B\left(x_{i}, R_{i}\right) \cap B(y, \beta s)=\emptyset \forall i<j\right] \\
& =\sum_{j \in J_{y}}\left(\prod_{i=1}^{j-1} \mathbb{P}\left[B\left(x_{i}, R_{i}\right) \cap B(y, \beta s)=\emptyset\right]\right) \mathbb{P}\left[B\left(x_{j}, R_{j}\right) \cap B(y, \beta s) \neq \emptyset\right] .
\end{aligned}
$$

Now, by the definition of the partition $P_{R_{1}, \ldots, R_{n}}^{x_{1}, \ldots, x_{n}}$ we have

$$
\left\{B(y, \beta s) \not P_{R_{1}, \ldots, R_{n}}^{x_{1}, \ldots, x_{n}}(x)\right\}=\bigcup_{j \in J_{y}} A_{j} .
$$

Thus, using the independence of $R_{1}, \ldots R_{n}$ once more,

$$
\begin{array}{cc}
1- & \mathbb{P}\left[B(y, \beta s) \subseteq P_{R_{1}, \ldots, R_{n}}^{x_{1}, \ldots, x_{n}}(x)\right] \leqslant \sum_{j \in J_{y}} \mathbb{P}\left[A_{j}\right] \\
= & \sum_{j \in J_{y}}\left(\prod_{i=1}^{j-1} \mathbb{P}\left[B\left(x_{i}, R_{i}\right) \cap B(y, \beta s)=\emptyset\right]\right) \\
& \cdot \mathbb{P}\left[B\left(x_{j}, R_{j}\right) \cap B(y, \beta s) \notin\{\emptyset, B(y, \beta s)\}\right] \\
\stackrel{(2.3) \wedge(2.8)}{\leqslant} & \left(1-K^{-32 \beta}\right)+\left(1-K^{-32 \beta}\right) \frac{\left|J_{y}\right|}{K^{4}-1} \\
\stackrel{(2.7)}{\leqslant} & \left(1-K^{-32 \beta}\right)\left(1+\frac{K^{3}}{K^{4}-1}\right) \\
\leqslant & 1-K^{-64 \beta},
\end{array}
$$


where in (2.9) we used the fact that, since $K \geqslant 2$ and $\beta<\frac{1}{40}$, we have

$$
\frac{K^{3}}{K^{4}-1} \leqslant K^{-32 \beta} .
$$

Indeed, this is equivalent to $32 \beta \leqslant \frac{\log \left(K-K^{-3}\right)}{\log K}$. But the function $K \mapsto \frac{\log \left(K-K^{-3}\right)}{\log K}$ is increasing on $(1, \infty)$, since its derivative is

$$
\frac{4}{\left(K^{5}-K\right) \log K}+\frac{\log \left(K^{4} /\left(K^{4}-1\right)\right)}{K(\log K)^{2}} \geqslant 0 .
$$

Thus it suffices to check that $32 \beta \leqslant \frac{\log \left(2-2^{-3}\right)}{\log 2}$, which is true since $\beta<\frac{1}{40}$.

\section{The random embedding}

Fix $\varepsilon \in(0,1 / 2), \theta \in(0,1)$ and $K \geqslant 2$. Write $K=e^{\gamma}$, and define

$$
N \stackrel{\text { def }}{=}\left\lceil\frac{c \gamma}{\theta}\right\rceil=\left\lceil\frac{c \log K}{\theta}\right\rceil
$$

where $c>0$ is a universal constant that will be determined later. It will also be convenient to write

$$
\tau \stackrel{\text { def }}{=} \frac{\varepsilon^{\theta}}{32 \gamma^{\theta}} .
$$

Let $(X, d)$ be a finite metric space whose doubling constant is at most $K$. By normalization assume that $\operatorname{diam}(X)=1$. For every $i \in \mathbb{N}$, let $\left\{x_{1}^{i}, \ldots, x_{n_{i}}^{i}\right\}$ be a $\frac{1}{4} \tau^{i /(1-\varepsilon)}$-net of $X$. For every $i, k \in \mathbb{N}$ and $j \in\left\{1, \ldots, n_{i}\right\}$, let $R_{i j}^{k}$ be a random variable whose density is $\phi_{s}$, as given in $(2.2)$, with $s=\tau^{i /(1-\varepsilon)}$. We will also use random variables $\left\{U_{i}^{k}(C): i, k \in \mathbb{N}, C \subseteq X\right\}$, each of which is uniformly distributed on the interval $[0,1]$ (thus for each $i, k \in \mathbb{N}$ we have $2^{|X|}$ such random variables). Throughout the argument below it is assumed that the random variables

$$
\left\{R_{i j}^{k}: i, k \in \mathbb{N}, j \in\left\{1, \ldots, n_{i}\right\}\right\} \bigcup\left\{U_{i}^{k}(C): i, k \in \mathbb{N}, C \subseteq X\right\}
$$

are mutually independent and defined on some probability space $(\Omega, \mathbb{P})$.

We will now consider the random partitions

$$
P_{i}^{k} \stackrel{\text { def }}{=} P_{R_{i 1}^{k}, \ldots, R_{i_{i}}^{k}}^{x_{i}^{i}, \ldots, x_{n_{i}}^{i}}
$$

where $P_{R_{i 1}^{k}, \ldots, R_{i n_{i}}^{k}}^{x_{1}, \ldots, x_{n_{i}}}$ is defined as in (2.1). For $i \in \mathbb{N}$ and $k \in\{1, \ldots, N\}$ define a random mapping $f_{i}^{k}: X \rightarrow \mathbb{R}$ by

$$
f_{i}^{k}(x) \stackrel{\text { def }}{=} U_{i}^{k}\left(P_{i}^{k}(x)\right) \cdot \min \left\{\tau^{i}, 64 \gamma \tau^{-\frac{i \varepsilon}{1-\varepsilon}-1} d\left(x, X \backslash P_{i}^{k}(x)\right)\right\} .
$$


Finally, we define a random embedding $F: X \rightarrow \mathbb{R}^{N}$ as follows:

$$
F(x)=\left(\frac{\sum_{i=1}^{\infty} f_{i}^{1}(x)}{\sqrt{N}}, \ldots, \frac{\sum_{i=1}^{\infty} f_{i}^{N}(x)}{\sqrt{N}}\right) \in \mathbb{R}^{N} .
$$

Note that, by the definition of $f_{i}^{k}$, the sums appearing in (3.6) converge geometrically.

Although $F$ is random, it satisfies the desired $(1-\varepsilon)$-Hölder condition deterministically. The randomness will enter when we prove that with positive probability $\|F(x)-F(y)\|_{2}$ satisfies the desired lower bound for all $x, y \in X$.

Lemma 3.1. For every $x, y \in X$ we have

$$
\begin{aligned}
\|F(x)-F(y)\|_{2} & \leqslant \max _{k \in\{1, \ldots, N\}} \sum_{i=1}^{\infty}\left|f_{i}^{k}(x)-f_{i}^{k}(y)\right| \\
& \lesssim \frac{\gamma^{(1+\theta)(1-\varepsilon)}}{\varepsilon^{1+\theta}} d(x, y)^{1-\varepsilon} \lesssim\left(\frac{\log K}{\varepsilon}\right)^{1+\theta} d(x, y)^{1-\varepsilon} .
\end{aligned}
$$

Proof. We first claim that for all $i \in \mathbb{N}$ and $k \in\{1, \ldots, N\}$ we have

$$
\left|f_{i}^{k}(x)-f_{i}^{k}(y)\right| \leqslant \min \left\{\tau^{i}, 64 \gamma \tau^{-\frac{i \varepsilon}{1-\varepsilon}-1} d(x, y)\right\}
$$

To verify (3.8) we may assume without loss of generality that $f_{i}^{k}(x)>f_{i}^{k}(y)$. If $P_{i}^{k}(x) \neq P_{i}^{k}(y)$, then

$$
f_{i}^{k}(x)-f_{i}^{k}(y) \leqslant f_{i}^{k}(x) \leqslant \min \left\{\tau^{i}, 64 \gamma \tau^{-\frac{i \varepsilon}{1-\varepsilon}-1} d\left(x, X \backslash P_{i}^{k}(x)\right)\right\},
$$

which is trivially bounded from above by the right hand side of (3.8) since we have $y \in X \backslash P_{i}^{k}(x)$. If $P_{i}^{k}(x)=P_{i}^{k}(y)=C$, then it cannot be the case that $f_{i}^{k}(y)=U_{i}^{k}(C) \tau^{i}$, since otherwise $f_{i}^{k}(x) \leqslant f_{i}^{k}(y)$, contrary to our assumption. We therefore necessarily have

$$
\begin{aligned}
& f_{i}^{k}(x)-f_{i}^{k}(y)=f_{i}^{k}(x)-64 U_{i}^{k}(C) \gamma \tau^{-\frac{i \varepsilon}{1-\varepsilon}-1} d(y, X \backslash C) \\
& \leqslant 64 U_{i}^{k}(C) \gamma \tau^{-\frac{i \varepsilon}{1-\varepsilon}-1}(d(x, X \backslash C)-d(y, X \backslash C)) \leqslant 64 \gamma \tau^{-\frac{i \varepsilon}{1-\varepsilon}-1} d(x, y)
\end{aligned}
$$

Since, by the definition $(3.5), f_{i}^{k}(x), f_{i}^{k}(y) \in\left[0, \tau^{i}\right]$, we also have $f_{i}^{k}(x)-f_{i}^{k}(y) \leqslant \tau^{i}$. This, in conjunction with (3.9), concludes the proof of (3.8).

The first inequality of (3.7) is an immediate consequence of the definition (3.6). As $K=e^{\gamma}$, the third inequality in (3.7) is a trivial overestimate. Note also that since for all $z \in X$ we have $\|F(z)\|_{2} \leqslant \sum_{i=1}^{\infty} \tau^{i} \lesssim \tau$, the bound in the second inequality of (3.7) holds true if $d(x, y)>\tau^{1+\frac{1}{1-\varepsilon}} /(64 \gamma)$. We may therefore assume that $d(x, y) \leqslant \tau^{1+\frac{1}{1-\varepsilon}} /(64 \gamma)$. Let $m \in \mathbb{N}$ be the integer satisfying

$$
\frac{\tau}{64 \gamma} \cdot \tau^{\frac{m+1}{1-\varepsilon}}<d(x, y) \leqslant \frac{\tau}{64 \gamma} \cdot \tau^{\frac{m}{1-\varepsilon}}
$$


Then

$$
\begin{aligned}
\|F(x)-F(y)\|_{2} & \leqslant\left(\frac{1}{N} \sum_{k=1}^{N}\left(\sum_{i=1}^{\infty}\left|f_{i}^{k}(x)-f_{i}^{k}(y)\right|\right)^{2}\right)^{1 / 2} \\
& \leqslant \max _{k \in\{1, \ldots, N\}} \sum_{i=1}^{\infty}\left|f_{i}^{k}(x)-f_{i}^{k}(y)\right| \\
& \stackrel{(3.8)}{\lesssim} \frac{\gamma}{\tau} d(x, y) \sum_{i=1}^{m} \tau^{-\frac{i \varepsilon}{1-\varepsilon}}+\sum_{i=m+1}^{\infty} \tau^{i} \\
& \stackrel{(3.2)}{\lesssim} \frac{\gamma^{1+\theta}}{\varepsilon^{\theta}} d(x, y) \sum_{i=1}^{m} \tau^{-\frac{i \varepsilon}{1-\varepsilon}}+\sum_{i=m+1}^{\infty} \tau^{i} .
\end{aligned}
$$

We estimate the two sums in (3.11) separately (recalling that $0<\varepsilon, \tau<1 / 2$ ):

$$
\sum_{i=1}^{m} \tau^{-\frac{i \varepsilon}{1-\varepsilon}}=\frac{\tau^{-\frac{m \varepsilon}{1-\varepsilon}}-1}{1-\tau^{\frac{\varepsilon}{1-\varepsilon}}} \lesssim \frac{1}{\varepsilon} \tau^{-\frac{m \varepsilon}{1-\varepsilon}} \stackrel{(3.10)}{\lesssim} \frac{\tau^{\varepsilon}}{\varepsilon \gamma^{\varepsilon} d(x, y)^{\varepsilon}} \stackrel{(3.2)}{\lesssim} \frac{1}{\varepsilon \gamma^{(1+\theta) \varepsilon} d(x, y)^{\varepsilon}}
$$

Similarly,

$$
\sum_{i=m+1}^{\infty} \tau^{i}=\frac{\tau^{m+1}}{1-\tau} \stackrel{(3.10)}{\lesssim} \frac{\gamma^{1-\varepsilon}}{\tau^{1-\varepsilon}} d(x, y)^{1-\varepsilon} \stackrel{(3.2)}{\lesssim} \frac{\gamma^{(1+\theta)(1-\varepsilon)}}{\varepsilon^{\theta}} d(x, y)^{1-\varepsilon} .
$$

The desired bound (3.7) follows from substituting (3.12) and (3.13) into (3.11).

\subsection{The Hölder lower bound holds with positive probability}

For every $i \in \mathbb{N}$ write

$$
\delta_{i} \stackrel{\text { def }}{=} \tau^{\frac{i+2}{1-\varepsilon}}\left(\frac{4 \varepsilon}{c^{*} \gamma}\right)^{\frac{1}{1-\varepsilon}}
$$

where $c^{*}$ is the implied universal constant in the final inequality of (3.7). Let $\mathscr{N}_{i}$ be a $\delta_{i}$-net of $X$.

Consider the following set:

$$
M \stackrel{\text { def }}{=}\left\{(i, u, v) \in \mathbb{N} \times \mathscr{N}_{i} \times \mathscr{N}_{i}: \tau^{\frac{i}{1-\varepsilon}}<d(u, v) \leqslant 3 \tau^{\frac{i-1}{1-\varepsilon}}\right\} .
$$

For every $(i, u, v) \in M$ define $G(i, u, v) \subseteq\{1, \ldots, N\}$ as follows:

$$
G(i, u, v) \stackrel{\text { def }}{=}\left\{k \in\{1, \ldots, N\}:\left|\sum_{j=1}^{\infty} f_{j}^{k}(u)-\sum_{j=1}^{\infty} f_{j}^{k}(v)\right| \geqslant \frac{\tau^{i+1}}{2}\right\} .
$$

For every $(i, u, v) \in M$ let $E(i, u, v) \subseteq \Omega$ be the following event:

$$
E(i, u, v) \stackrel{\text { def }}{=}\left\{|G(i, u, v)| \geqslant \frac{N}{2}\right\}
$$


and consider the event $E \subseteq \Omega$ given by:

$$
E \stackrel{\text { def }}{=} \bigcap_{(i, u, v) \in M} E(i, u, v) \text {. }
$$

The relevance of the event $E$ is explained in the following lemma.

Lemma 3.2. If the event $E$ occurs then for all $x, y \in X$ we have

$$
\|F(x)-F(y)\|_{2} \gtrsim\left(\frac{\varepsilon}{\log K}\right)^{2 \theta} d(x, y)^{1-\varepsilon} .
$$

Proof. Let $i$ be the integer such that

$$
\tau^{\frac{i}{1-\varepsilon}}<d(x, y) \leqslant \tau^{\frac{i-1}{1-\varepsilon}}
$$

Since $\mathscr{N}_{i}$ is a $\delta_{i}$-net, where $\delta_{i}$ is given in (3.14), there exist $u, v \in \mathscr{N}_{i}$ such that

$$
\max \{d(u, x), d(v, y)\} \leqslant \tau^{\frac{i+2}{1-\varepsilon}}\left(\frac{4 \varepsilon}{c^{*} \gamma}\right)^{\frac{1}{1-\varepsilon}} .
$$

Assume that $k \in G(i, u, v)$. By Lemma 3.1 we have

$$
\begin{aligned}
& \max \left\{\left|\sum_{j=1}^{\infty} f_{j}^{k}(u)-\sum_{j=1}^{\infty} f_{j}^{k}(x)\right|,\left|\sum_{j=1}^{\infty} f_{j}^{k}(v)-\sum_{j=1}^{\infty} f_{j}^{k}(y)\right|\right\} \\
& \stackrel{(3.7) \wedge(3.20)}{\leqslant} c^{*}\left(\frac{\gamma}{\varepsilon}\right)^{1+\theta} \frac{4 \tau^{i+2} \varepsilon}{c^{*} \gamma} \stackrel{(3.2)}{=} \frac{\tau^{i+1}}{8}
\end{aligned}
$$

Since $k \in G(i, u, v)$ it follows that

$$
\left|\sum_{j=1}^{\infty} f_{j}^{k}(x)-\sum_{j=1}^{\infty} f_{j}^{k}(y)\right| \stackrel{(3.16) \wedge(3.21)}{\geqslant} \frac{\tau^{i+1}}{2}-2 \cdot \frac{\tau^{i+1}}{8}=\frac{\tau^{i+1}}{4} .
$$

Since we are assuming that the event $E$ occurs, the lower bound (3.22) holds for at least $N / 2$ values of $k \in\{1, \ldots, N\}$. Thus, by the definition of $F$,

$$
\|F(x)-F(y)\|_{2} \gtrsim \tau^{i+1} \stackrel{(3.2) \wedge(3.19)}{\gtrsim}\left(\frac{\varepsilon}{\gamma}\right)^{2 \theta} d(x, y)^{1-\varepsilon} .
$$

Due to Lemma 3.1 and Lemma 3.2, Theorem 1.2 will be proven (with the bounds claimed in (1.2), with $\delta=3 \theta$ ), once we establish the following lemma.

Lemma 3.3. We have $\mathbb{P}[E]>0$, provided $c$ in (3.1) is a large enough universal constant.

The key tool used in the proof of Lemma 3.3 is the Lovász Local Lemma [11]. The variant of this lemma that is stated below is not the same as the classical formulation of the Lovász Local Lemma, but it is a consequence of it, as explained in [1], where a more general statement is needed. For more information on the Lovász Local Lemma and some of its striking applications, see for example the survey of Alon [2]. 
Lemma 3.4 (Lovász Local Lemma). Fix $q \in(0,1)$ and $d \in \mathbb{N}$. Let $\mathcal{A}_{1}, \mathcal{A}_{2}, \ldots \mathcal{A}_{n}$ be measurable sets in some probability space $(\Omega, \mathbb{P})$. Let $G=\left(V, E_{G}\right)$ be a graph on the vertex set $V=\left\{\mathcal{A}_{1}, \mathcal{A}_{2}, \ldots \mathcal{A}_{n}\right\}$ with maximal degree $d$. Let

$$
\rho:\left\{\mathcal{A}_{1}, \mathcal{A}_{2}, \ldots \mathcal{A}_{n}\right\} \rightarrow \mathbb{N}
$$

be a mapping that satisfies the condition

$$
\left\{\mathcal{A}_{i}, \mathcal{A}_{j}\right\} \in E_{G} \Longrightarrow \rho\left(\mathcal{A}_{i}\right)=\rho\left(\mathcal{A}_{j}\right) .
$$

Assume that for any $i \in\{1, \ldots, n\}$ we have

$$
\mathbb{P}\left[\bigcap_{j \in Q}\left(\Omega \backslash \mathcal{A}_{j}\right) \cap \mathcal{A}_{i}\right] \leqslant q \mathbb{P}\left[\bigcap_{j \in Q}\left(\Omega \backslash \mathcal{A}_{j}\right)\right]
$$

for all

$$
Q \subseteq\left\{j \in\{1, \ldots, n\}:\left\{\mathcal{A}_{i}, \mathcal{A}_{j}\right\} \notin E_{G} \wedge \rho\left(\mathcal{A}_{i}\right) \geqslant \rho\left(\mathcal{A}_{j}\right)\right\} .
$$

Assume also that

$$
e q(d+1) \leqslant 1 .
$$

Then

$$
\mathbb{P}\left[\bigcap_{i=1}^{n}\left(\Omega \backslash \mathcal{A}_{i}\right)\right]>0
$$

To use Lemma 3.4 we proceed as follows. For $(i, u, v) \in M$ consider the following random subset of $\{1, \ldots, k\}$ :

$$
L(i, u, v) \stackrel{\text { def }}{=}\left\{k \in\{1, \ldots, N\}:\left|\sum_{j=1}^{i} f_{j}^{k}(u)-\sum_{j=1}^{i} f_{j}^{k}(v)\right| \geqslant 2 \tau^{i+1}\right\} .
$$

For $(i, u, v) \in M$ and $k \in\{1, \ldots, N\}$ define the following event:

$$
S(i, u, v, k) \stackrel{\text { def }}{=}\{k \in L(i, u, v)\} .
$$

Finally, we also define the following event for all $(i, u, v) \in M$ :

$$
T(i, u, v) \stackrel{\text { def }}{=}\left\{|L(i, u, v)| \geqslant \frac{N}{2}\right\} .
$$

Lemma 3.5. For all $(i, u, v) \in M$ we have $T(i, u, v) \subseteq E(i, u, v)$.

Proof. Using (3.8) we see that for all $k \in\{1, \ldots, N\}$,

$$
\left|\sum_{j=i+1}^{\infty} f_{j}^{k}(u)-\sum_{j=i+1}^{\infty} f_{j}^{k}(v)\right| \leqslant \sum_{j=i+1}^{\infty} \tau^{j} \leqslant \frac{3}{2} \tau^{i+1} .
$$


Hence, if $k \in L(i, u, v)$ then

$$
\begin{aligned}
\left|\sum_{j=1}^{\infty} f_{j}^{k}(u)-\sum_{j=1}^{\infty} f_{j}^{k}(v)\right| & \geqslant\left|\sum_{j=1}^{i} f_{j}^{k}(u)-\sum_{j=1}^{i} f_{j}^{k}(v)\right|-\left|\sum_{j=i+1}^{\infty} f_{j}^{k}(u)-\sum_{j=i+1}^{\infty} f_{j}^{k}(v)\right| \\
(3.24) & \wedge(3.27) \\
& \frac{\tau^{i+1}}{2} .
\end{aligned}
$$

This means that $L(i, u, v) \subseteq G(i, u, v)$, and hence

$$
|L(i, u, v)| \geqslant \frac{N}{2} \Longrightarrow|G(i, u, v)| \geqslant \frac{N}{2} .
$$

Before proceeding with the proof of Lemma 3.3, it is beneficial for us to introduce some notation related to the random partitions that are used in the definition of the embedding $F$. For $i \in \mathbb{N}$ and $k \in\{1, \ldots, N\}$ the partition $P_{i}^{k}$ was defined in (3.4), where $\left\{x_{1}^{i}, \ldots, x_{n_{i}}^{i}\right\}$ is a fixed $\frac{1}{4} \tau^{i /(1-\varepsilon)}$-net of $X$, and $R_{i 1}^{k}, \ldots, R_{i n_{i}}^{k}$ are i.i.d. random variables whose density is $\phi_{s}$ as given in (2.2), with $s=\tau^{i /(1-\varepsilon)}$. For every $y \in X$ define

$$
J(i, y) \stackrel{\text { def }}{=}\left\{j \in\left\{1, \ldots, n_{i}\right\}: d\left(y, x_{j}^{i}\right) \leqslant 2 \tau^{\frac{i}{1-\varepsilon}}\right\} .
$$

We will consider the following random variable:

$$
j(i, k, y) \stackrel{\text { def }}{=} \min \left\{j \in J(i, y): y \in B\left(x_{j}^{i}, R_{i j}^{k}\right)\right\} .
$$

To see that $j(i, k, y)$ is well defined, note that since $\left\{x_{1}^{i}, \ldots, x_{n_{i}}^{i}\right\}$ is a $\frac{1}{4} \tau^{i /(1-\varepsilon)}$-net of $X$ and $R_{i 1}^{k}, \ldots, R_{i n_{i}}^{k} \geqslant \frac{1}{4} \tau^{i /(1-\varepsilon)}$, there must be some $j \in\{1, \ldots, N\}$ for which $y \in B\left(x_{j}^{i}, R_{i j}^{k}\right)$, and since $R_{i j}^{k} \leqslant \frac{1}{2} \tau^{i /(1-\varepsilon)}$ necessarily $j \in J(i, y)$.

From the definition (2.1) we see that

$$
P_{i}^{k}(y)=B\left(x_{j(i, k, y)}^{i}, R_{i j(i, k, y)}^{k}\right) \backslash \bigcup_{\ell=1}^{j(i, k, y)-1} B\left(x_{\ell}^{i}, R_{i \ell}^{k}\right) .
$$

But note that if there exists $z \in B\left(x_{\ell}^{i}, R_{i \ell}^{k}\right) \cap B\left(x_{j(i, k, y)}^{i}, R_{i j(i, k, y)}^{k}\right)$ then

$$
d\left(x_{\ell}^{i}, y\right) \leqslant d\left(x_{\ell}^{i}, z\right)+d\left(z, x_{j(i, k, y)}^{i}\right)+d\left(x_{j(i, k, y)}^{i}, y\right) \leqslant R_{i \ell}^{k}+2 R_{i j(i, k, y)}^{k} \leqslant 2 \tau^{\frac{i}{1-\varepsilon}},
$$

implying that $\ell \in J(i, y)$. It follows from this that (3.30) can be rewritten as follows:

$$
P_{i}^{k}(y)=B\left(x_{j(i, k, y)}^{i}, R_{i j(i, k, y)}^{k}\right) \backslash \underset{\ell \in J(i, y) \cap\{1, \ldots, j(i, k, y)-1\}}{\bigcup} B\left(x_{\ell}^{i}, R_{i \ell}^{k}\right) .
$$

To continue with our plan to use Lemma 3.4, we define a graph $H=\left(V, E_{H}\right)$, where $V \stackrel{\text { def }}{=}\{T(i, u, v):(i, u, v) \in M\}$, and a mapping $\rho: V \rightarrow \mathbb{N}$, as follows:

$$
\begin{aligned}
\left\{T(i, u, v), T\left(i^{\prime}, u^{\prime}, v^{\prime}\right)\right\} \in E_{H} \Longleftrightarrow i & =i^{\prime} \wedge d\left(\{u, v\},\left\{u^{\prime}, v^{\prime}\right\}\right) \leqslant 4 \tau^{\frac{i}{1-\varepsilon}}, \\
\rho(T(i, u, v)) & =i .
\end{aligned}
$$


Lemma 3.6. The maximal degree of $H$ is at most $K^{c^{* *}(\log \log K+\log (1 / \varepsilon))}$, where $c^{* *} \in(0, \infty)$ is a universal constant.

Proof. Given $(i, u, v) \in M$, we need to bound the number of $\left(i, u^{\prime}, v^{\prime}\right) \in M$ satisfying $d\left(\{u, v\},\left\{u^{\prime}, v^{\prime}\right\}\right) \leqslant 4 \tau^{i /(1-\varepsilon)}$. We may assume that $d\left(u, u^{\prime}\right) \leqslant 4 \tau^{i /(1-\varepsilon)}$. Recall that from the definition of $\mathrm{M}$ in (3.15) we know that $d(u, v), d\left(u^{\prime}, v^{\prime}\right) \leqslant$ $3 \tau^{(i-1) /(1-\varepsilon)}$. Hence the points $v, u^{\prime}, v^{\prime}$ are all in the ball $B$ of radius $r=4 \tau^{(i-1) /(1-\varepsilon)}$ centered at $u$, implying that the number of $\left(i, u^{\prime}, v^{\prime}\right)$ as above is at most $\left|B \cap \mathscr{N}_{i}\right|^{2}$. Since $(X, d)$ is $K$-doubling, $B$ can be covered by at most $K^{1+\log _{2}\left(2 r / \delta_{i}\right)}$ balls of radius $\delta_{i} / 2$, each of which contains at most one point from the $\delta_{i}$-net $\mathscr{N}_{i}$ (recall (3.14) for the definition of $\delta_{i}$ ). Hence, the maximal degree of $H$ is at most

$$
K^{4+2 \log _{2}\left(r / \delta_{i}\right)}=K^{O(\log \log K+\log (1 / \varepsilon))} .
$$

Lemma 3.7. For every $(i, u, v) \in M$ and for every

$$
Q \subseteq\left\{\left(i^{\prime}, u^{\prime}, v^{\prime}\right) \in M: i \geqslant i^{\prime} \wedge\left\{T(i, u, v), T\left(i^{\prime}, u^{\prime}, v^{\prime}\right)\right\} \notin E_{H}\right\}
$$

we have

$$
\begin{aligned}
& \mathbb{P}\left[\bigcap_{\left(i^{\prime}, u^{\prime}, v^{\prime}\right) \in Q} T\left(i^{\prime}, v^{\prime}, u^{\prime}\right) \backslash T(i, u, v)\right] \\
& \quad \leqslant\left(\frac{\varepsilon}{\log K}\right)^{\theta N / 2} \mathbb{P}\left[\bigcap_{\left(i^{\prime}, u^{\prime}, v^{\prime}\right) \in Q} T\left(i^{\prime}, v^{\prime}, u^{\prime}\right)\right] .
\end{aligned}
$$

Proof. Denote

$$
W \stackrel{\text { def }}{=} \bigcap_{\left(i^{\prime}, u^{\prime}, v^{\prime}\right) \in Q} T\left(i^{\prime}, v^{\prime}, u^{\prime}\right) .
$$

Consider the following subsets $\mathscr{X}, \mathscr{Y}$ of the random variables given in (3.3):

$$
\begin{aligned}
& \mathscr{X} \stackrel{\text { def }}{=}\left\{R_{i^{\prime} j}^{k}: i^{\prime} \in\right.\left.\{1, \ldots, i-1\}, j \in\left\{1, \ldots, n_{i^{\prime}}\right\}, k \in\{1, \ldots, N\}\right\} \\
& \cup\left\{U_{i^{\prime}}^{k}(C): i^{\prime} \in\{1, \ldots, i-1\}, k \in\{1, \ldots, N\}, C \subseteq X\right\}, \\
& \mathscr{Y} \stackrel{\text { def }}{=}\left\{R_{i j}^{k}: j \in\left\{1, \ldots, n_{i}\right\} \backslash J(i, u), k \in\{1, \ldots, N\}\right\} \\
& \bigcup\left\{U_{i}^{k}(C): k \in\{1, \ldots, N\}, C \subseteq X \backslash B\left(u, 2 \tau^{i /(1-\varepsilon)}\right)\right\} .
\end{aligned}
$$

The event $W$ depends only on the variables $\mathscr{X} \cup \mathscr{Y}$. Indeed, for $\left(i^{\prime}, u^{\prime}, v^{\prime}\right) \in Q$ with $i^{\prime}<i$, it follows from the definitions (3.26), (3.24), (3.5) that the event $T\left(i^{\prime}, u^{\prime}, v^{\prime}\right)$ depends only on the variables $\mathscr{X}$. If $\left(i, u^{\prime}, v^{\prime}\right) \in Q$ and $\left\{T(i, u, v), T\left(i, u^{\prime}, v^{\prime}\right)\right\} \notin E_{H}$ then it follows from $(3.32)$ that $d\left(u^{\prime}, u\right), d\left(v^{\prime}, u\right)>4 \tau^{i /(1-\varepsilon)}$. Since the diameters of $P_{i}^{k}\left(u^{\prime}\right)$ and $P_{i}^{k}\left(v^{\prime}\right)$ are at most $\tau^{i /(1-\varepsilon)}$, it follows that

$$
P_{i}^{k}\left(u^{\prime}\right), P_{i}^{k}\left(v^{\prime}\right) \subseteq X \backslash B\left(u, 2 \tau^{i /(1-\varepsilon)}\right),
$$


and hence $U_{i}^{k}\left(P_{i}^{k}\left(u^{\prime}\right)\right), U_{i}^{k}\left(P_{i}^{k}\left(v^{\prime}\right)\right) \in \mathscr{Y}$. Similarly, $J(i, u) \cap J\left(i, u^{\prime}\right)=\emptyset$ and $J(i, u) \cap J\left(i, v^{\prime}\right)=\emptyset$, and hence from the identity (3.31) we know that $P_{i}^{k}\left(u^{\prime}\right), P_{i}^{k}\left(v^{\prime}\right)$ depend only on the variables $\mathscr{Y}$. These observations, combined with the definition (3.5), imply that $f_{i}^{k}\left(u^{\prime}\right), f_{i}^{k}\left(v^{\prime}\right)$ depend only on the variables $\mathscr{Y}$, and from the definitions (3.26) and (3.24) we conclude that the event $T\left(i, u^{\prime}, v^{\prime}\right)$ depends only on the variables $\mathscr{X} \cup \mathscr{Y}$, as required.

Recalling the definitions (3.24), (3.25), and (3.26), it follows from the above argument that

$$
\mathbb{P}[W \cap T(i, u, v)]=\int_{W} \mathbb{P}\left[\sum_{k=1}^{N} \mathbf{1}_{S(i, u, v, k)} \geqslant \frac{N}{2} \mid \mathscr{X} \cup \mathscr{Y}\right] d \mathbb{P} .
$$

To estimate the right hand side of $(3.36)$, for each $k \in\{1, \ldots, n\}$ consider the event

$$
Z_{k} \stackrel{\text { def }}{=} S(i, u, v, k) \cap\left\{B\left(u, \frac{\tau}{64 \gamma} \tau^{\frac{i}{1-\varepsilon}}\right) \subseteq P_{i}^{k}(u)\right\} .
$$

From (3.36) we then see that

$$
\mathbb{P}[W \cap T(i, u, v)] \geqslant \int_{W} \mathbb{P}\left[\sum_{k=1}^{N} \mathbf{1}_{Z_{k}} \geqslant \frac{N}{2} \mid \mathscr{X} \cup \mathscr{Y}\right] d \mathbb{P} .
$$

An application of Lemma 2.2 with $\beta=\frac{\tau}{64 \gamma}$ yields the estimate

$$
\mathbb{P}\left[B\left(u, \frac{\tau}{64 \gamma} \tau^{\frac{i}{1-\varepsilon}}\right) \subseteq P_{i}^{k}(u)\right] \geqslant K^{-\tau / \gamma}=e^{-\tau} .
$$

Moreover, it follows from the definition (3.5) that if $B\left(u, \frac{\tau}{64 \gamma} \tau^{\frac{i}{1-\varepsilon}}\right) \subseteq P_{i}^{k}(u)$ then we have $f_{i}^{k}(u)=U_{i}^{k}\left(P_{i}^{k}(u)\right) \tau^{i}$. Hence, recalling the definition (3.25), we see that

$$
\begin{aligned}
Z_{k}=\{B & \left.\left(u, \frac{\tau}{64 \gamma} \tau^{\frac{i}{1-\varepsilon}}\right) \subseteq P_{i}^{k}(u)\right\} \\
& \left.\bigcap\left|\sum_{j=1}^{i-1}\left(f_{j}^{k}(u)-f_{j}^{k}(v)\right)+U_{i}^{k}\left(P_{i}^{k}(u)\right) \tau^{i}-f_{i}^{k}(v)\right| \geqslant 2 \tau^{i+1}\right\} .
\end{aligned}
$$

From the identity (3.31) we see that the event $\left\{B\left(u, \frac{\tau}{64 \gamma} \tau^{\frac{i}{1-\varepsilon}}\right) \subseteq P_{i}^{k}(u)\right\}$ is independent of $\mathscr{X} \cup \mathscr{Y}$. Thus, denoting $a \stackrel{\text { def }}{=} \tau^{-i} \sum_{j=1}^{i-1}\left(f_{j}^{k}(u)-f_{j}^{k}(v)\right)-\tau^{-i} f_{i}^{k}(v)$, we have

$$
\begin{aligned}
p \stackrel{\text { def }}{=} & \mathbb{P}\left[Z_{k} \mid \mathscr{X} \cup \mathscr{Y}\right] \\
\stackrel{(3.40)}{=} & \mathbb{P}\left[B\left(u, \frac{\tau}{64 \gamma} \tau^{\frac{i}{1-\varepsilon}}\right) \subseteq P_{i}^{k}(u)\right] \\
& \quad \cdot \mathbb{P}\left[U_{i}^{k}\left(P_{i}^{k}(u)\right) \notin(a-2 \tau, a+2 \tau) \mid \mathscr{X} \cup \mathscr{Y}\right] \\
\stackrel{(3.39)}{\geqslant} & e^{-\tau}(1-4 \tau) \\
\geqslant & 1-5 \tau,
\end{aligned}
$$


where in (3.42) we used the fact that since $d(u, v)>\tau^{i /(1-\varepsilon)}$ (by the definition $(3.15)$ of $M)$, and $\operatorname{diam}\left(P_{i}^{k}(u)\right) \leqslant \tau^{i /(1-\varepsilon)}$, we have $P_{i}^{k}(u) \neq P_{i}^{k}(v)$, and therefore the random variable $U_{i}^{k}\left(P_{i}^{k}(u)\right)$, which is uniformly distributed on $[0,1]$, is independent of $a$ and $\mathscr{X} \cup \mathscr{Y}$.

Since after fixing the values of $\mathscr{X} \cup \mathscr{Y}$, the events $Z_{1}, \ldots, Z_{N}$ are independent, the Chernoff bound (see Theorem A.1.12 in [3]) implies that

$$
\begin{aligned}
\mathbb{P}\left[\sum_{k=1}^{N} \mathbf{1}_{Z_{k}} \geqslant \frac{N}{2} \mid \mathscr{X} \cup \mathscr{Y}\right] & =1-\mathbb{P}\left[\sum_{k=1}^{N} \mathbf{1}_{\Omega \backslash Z_{k}}>\frac{N}{2} \mid \mathscr{X} \cup \mathscr{Y}\right] \\
& \geqslant 1-\left(e^{p-\frac{1}{2}} \sqrt{2(1-p)}\right)^{N} \stackrel{(3.43)}{\geqslant} 1-(10 e \tau)^{N / 2}
\end{aligned}
$$

Substituting (3.44) into (3.38) shows that

$$
\mathbb{P}[W \cap T(i, u, v)] \geqslant\left(1-(30 \tau)^{N / 2}\right) \mathbb{P}[W] \stackrel{(3.2)}{\geqslant}\left(1-\left(\frac{\varepsilon}{\log K}\right)^{\theta N / 2}\right) \mathbb{P}[W],
$$

which is the same statement as (3.35).

Proof of Lemma 3.3. By Lemma 3.5 we have

$$
\mathbb{P}[E] \geqslant \mathbb{P}\left[\bigcap_{(i, u, v) \in M} T(i, u, v)\right] .
$$

Hence, due to Lemma 3.6 and Lemma 3.7, Lemma 3.3 will follow from Lemma 3.4 if the condition corresponding to (3.23) holds, i.e.,

$$
e\left(\frac{\varepsilon}{\log K}\right)^{\theta N / 2}\left(K^{O(\log \log K+\log (1 / \varepsilon))}+1\right) \leqslant 1 .
$$

This holds provided the constant $c$ in the definition (3.1) of $N$ is large enough.

\section{Snowflakes of the Heisenberg group}

As promised in the introduction, we first argue that a positive answer to the qualitative version of the Lang-Plaut question, as appearing in Question 1, implies its quantitative variant, i.e., that for every $K>0$ there is $N=N(K) \in \mathbb{N}$ and $D=D(K) \in(1, \infty)$ such that if $X \subseteq \ell_{2}$ has doubling constant $K$ then $c_{\mathbb{R}^{N}}(X) \leqslant D$. Indeed, if not then there would be some $K>0$ and a sequence $\left\{X_{n}\right\}_{n=1}^{\infty}$ of subsets of $\ell_{2}$ with doubling constant $K$ and satisfying $c_{\mathbb{R}^{n}}\left(X_{n}\right)>n$. By (1.1) there are finite subsets $F_{n} \subseteq X_{n}$ with $c_{\mathbb{R}^{n}}\left(F_{n}\right)>n$, and by translation and rescaling we may assume that $0 \in F_{n}$ and that $F_{n}$ is contained in the ball of $\ell_{2}$ centered at 0 of radius 1 . Let $Y \subseteq \ell_{2} \times \mathbb{R}$ be given by $Y=\bigcup_{n=1}^{\infty} F_{n} \times\left\{4^{n}\right\}$. One checks that $Y$ has doubling constant $O(K)$, and clearly all the $F_{n}$ embed into $Y$ isometrically. By the assumed positive answer to the Lang-Plaut problem it follows that $c_{\mathbb{R}^{N}}\left(F_{n}\right) \leqslant D$ for some $N \in \mathbb{N}$ and $D \in(1, \infty)$, contradicting the fact that $c_{\mathbb{R}^{N}}\left(F_{n}\right)>n$ for $n \geqslant N$. 
Proof of Theorem 1.3. For $\theta>0$ define $\delta_{\theta}: \mathbb{H}_{n} \rightarrow \mathbb{H}_{n}$ by $\delta_{\theta}(z, t)=\left(\theta z, \theta^{2} t\right)$. Note that for every measurable $A \subseteq \mathbb{H}_{n}=\mathbb{C}^{n} \times \mathbb{R}$ we have $\operatorname{vol}\left(\delta_{\theta}(A)\right)=\theta^{2 n+2} \operatorname{vol}(A)$. For $p \in[1,2)$ and $(z, t) \in \mathbb{H}_{n}$ define

$$
M_{p}(z, t) \stackrel{\text { def }}{=} \sqrt[4]{|z|^{4}+t^{2}}\left(\cos \left(\frac{p}{2} \arccos \left(\frac{|z|^{2}}{\sqrt{|z|^{4}+t^{2}}}\right)\right)\right)^{1 / p} .
$$

It was shown in [22] that $M_{p}\left(x y^{-1}\right) \leqslant M_{p}(x)+M_{p}(y)$ for all $x, y \in \mathbb{H}_{n}$. Therefore $d_{M_{p}}(x, y)=M_{p}\left(y^{-1} x\right)$ is a left-invariant metric on $\mathbb{H}_{n}$. It was also shown in [22] that $\sqrt{1-\frac{p}{2}} N_{0}(x) \leqslant M_{p}(x) \leqslant N_{0}(x)$ for all $x \in \mathbb{H}_{n}$, and there exists $f: \mathbb{H}_{n} \rightarrow \ell_{2}$ satisfying $\|f(x)-f(y)\|=d_{M_{p}}(x, y)^{p / 2}$ for all $x, y \in \mathbb{H}_{n}$. Setting $p=2(1-\varepsilon)$, we see that for all distinct $x, y \in \mathbb{H}_{n}$ we have

$$
\frac{\|f(x)-f(y)\|}{d_{N_{0}}(x, y)^{1-\varepsilon}}=\left(\frac{d_{M_{p}}(x, y)}{d_{N_{0}}(x, y)}\right)^{p / 2} \in\left[\varepsilon^{(1-\varepsilon) / 2}, 1\right] \subseteq[\sqrt{\varepsilon}, 1] .
$$

For $x \in \mathbb{H}_{n}$ and $r>0$ denote $B_{p}(x, r)=\left\{y \in \mathbb{H}_{n}: d_{M_{p}}(x, y)^{p / 2} \leqslant r\right\}$. Note that $B_{p}(0, r)=\delta_{2^{2 / p}}\left(B_{p}(0, r / 2)\right)$, since for every $\theta>0$ and $x, y \in \mathbb{H}_{n}$ we have $d_{M_{p}}\left(\delta_{\theta}(x), \delta_{\theta}(y)\right)=\theta d_{M_{p}}(x, y)$. Hence, by left-invariance of $d_{M_{p}}^{p / 2}$ and the Lebesgue measure $\operatorname{vol}(\cdot)$, for all $x \in \mathbb{H}_{n}$ and $r>0$ we have

$$
\operatorname{vol}\left(B_{p}(x, r)\right)=2^{4(n+1) / p} \operatorname{vol}\left(B_{p}(x, r / 2)\right) .
$$

This implies that $\left(\mathbb{H}_{n}, d_{M_{p}}^{p / 2}\right)=\left(\mathbb{H}_{n}, d_{M_{p}}^{1-\varepsilon}\right)$, and hence also its isometric copy $f\left(\mathbb{H}_{n}\right) \subseteq \ell_{2}$ has doubling constant $2^{8(n+1) / p} \leqslant 2^{16(n+1)}$.

We end with the proof of the distortion lower bound (1.4). Suppose $f: \mathbb{H}_{1} \rightarrow \ell_{2}$ satisfies

$$
d_{N_{0}}(x, y)^{1-\varepsilon} \leqslant\|f(x)-f(y)\| \leqslant D d_{N_{0}}(x, y)^{1-\varepsilon} \quad \forall x, y \in \mathbb{H}_{1} .
$$

Our goal is to prove that $D \gtrsim 1 / \sqrt{\varepsilon}$. Write $a=(1,0) \in \mathbb{H}_{1}, b=(i, 0) \in \mathbb{H}_{1}$ and $c=a b a^{-1} b^{-1}=(0,-4)$. Writing

$$
B_{m}=\left\{(u+i v, t) \in \mathbb{H}_{1}: u, v, t \in \mathbb{Z} \wedge N_{0}(u+i v, t) \leqslant m\right\},
$$

it follows from [5] that there exists a universal constant $C>0$ such that, for all $m \in \mathbb{N}$, we have

$$
\sum_{x \in B_{m}} \sum_{k=1}^{m^{2}} \frac{\left\|f\left(x c^{k}\right)-f(x)\right\|^{2}}{k^{2}} \lesssim \sum_{x \in B_{C m}}\left(\|f(x a)-f(x)\|^{2}+\|f(x b)-f(x)\|^{2}\right)
$$

Note that for all $x \in \mathbb{H}_{1}$ we have $d_{N_{0}}(x a, x)=N_{0}(a)=1$ and $d_{N_{0}}(x b, x)=1$. Moreover, for all $k \in \mathbb{N}$ and $x \in \mathbb{H}_{1}$ we have

$$
d_{N_{0}}\left(x c^{k}, x\right)=N_{0}\left(c^{k}\right)=N_{0}(0,-4 k)=2 \sqrt{k} .
$$


Hence, using (4.1) and the fact that the cardinality of $B_{m}$ is bounded above and below by universal multiples of $m^{4}$, inequality (4.2) becomes

$$
D^{2} \gtrsim \sum_{k=1}^{m^{2}} \frac{N_{0}\left(c^{k}\right)^{2(1-\varepsilon)}}{k^{2}} \gtrsim \sum_{k=1}^{m^{2}} \frac{1}{k^{1+\varepsilon}} .
$$

Letting $m$ tend to $\infty$ we deduce that $D^{2} \gtrsim \sum_{k=1}^{\infty} \frac{1}{k^{1+\varepsilon}} \gtrsim \frac{1}{\varepsilon}$, as required.

\section{References}

[1] Abraham, I., Bartal, Y. and Neiman, O.: Embedding metric spaces in their intrinsic dimension. In Proceedings of the Nineteenth Annual ACM-SIAM Symposium on Discrete Algorithms, 363-372. ACM, New York, 2008.

[2] Alon, N.: Probabilistic proofs of existence of rare events. In Geometric aspects of functional analysis (1987-88), 186-201. Lecture Notes in Mathematics 1376, Springer, Berlin, 1989.

[3] Alon, N. And Spencer, J. H.: The probabilistic method. Wiley-Interscience Series in Discrete Mathematics and Optimization. John Wiley \& Sons, Hoboken, NJ, 2008.

[4] Assouad, P.: Plongements lipschitziens dans $\mathbb{R}^{n}$. Bull. Soc. Math. France 111 (1983), no. 4, 429-448.

[5] Austin, T., Naor, A. And Tessera, R.: Sharp quantitative nonembeddability of the Heisenberg group into superreflexive Banach spaces. Preprint available at http://www.cims.nyu.edu/ naor/homepage\%20files/UCHeis9.pdf, 2010. To appear in Groups Geom. Dyn.

[6] Bartal, Y., Recht, B. and Schulman, L. J.: Dimensionality reduction: beyond the Johnson-Lindenstrauss bound. In Proceedings of the 22nd Annual ACM-SIAM Symposium on Discrete Algorithms, 868-887. SIAM, Philadelphia, PA, 2011.

[7] Bourdon, M. And PAjot, H.: Poincaré inequalities and quasiconformal structure on the boundary of some hyperbolic buildings. Proc. Amer. Math. Soc. 127 (1999), no. 8, 2315-2324.

[8] Cheeger, J.: Differentiability of Lipschitz functions on metric measure spaces. Geom. Funct. Anal. 9 (1999), no. 3, 428-517.

[9] Cygan, J.: Subadditivity of homogeneous norms on certain nilpotent Lie groups. Proc. Amer. Math. Soc. 83 (1981), no. 1, 69-70.

[10] David, G. And Toro, T.: Reifenberg flat metric spaces, snowballs, and embeddings. Math. Ann. 315 (1999), no. 4, 641-710.

[11] ERdős, P. AND LovÁsz, L.: Problems and results on 3-chromatic hypergraphs and some related questions. In Infinite and finite sets (Colloq., Keszthely, 1973; dedicated to P. Erdős on his 60th birthday), Vol. II, 609-627. Colloq. Math. Soc. János Bolyai 10, North-Holland, Amsterdam, 1975.

[12] Gottlieb, A. And Krauthgamer, R.: A nonlinear approach to dimension reduction. In Proceedings of the 22nd Annual ACM-SIAM Symposium on Discrete Algorithms, 888-899. SIAM, Philadelphia, PA, 2011.

[13] Gupta, A., Krauthgamer, R. And Lee, J. R.: Bounded geometries, fractals, and low-distortion embeddings. In 44th Symposium on Foundations of Computer Science, 534-543. IEEE Computer Society, 2003. 
[14] Har-Peled, S. And Mendel, M.: Fast construction of nets in low-dimensional metrics and their applications. SIAM J. Comput. 35 (2006), no. 5, 1148-1184.

[15] Heinonen, J.: Lectures on analysis on metric spaces. Universitext. Springer-Verlag, New York, 2001.

[16] Heinonen, J.: Geometric embeddings of metric spaces. Report. University of Jyväskylä Dept. of Math. and Stat. 90, University of Jyväskylä, Jyväskylä, 2003.

[17] Kahane, J. P.: Hélices et quasi-hélices. In Mathematical analysis and applications, Part B, 417-433. Adv. in Math. Suppl. Stud. 7b, Academic Press, New YorkLondon, 1981.

[18] Korányi, A. and Reimann, H. M.: Quasiconformal mappings on the Heisenberg group. Invent. Math. 80 (1985), no. 2, 309-338.

[19] LaAkso, T. J.: Plane with $A_{\infty}$-weighted metric not bi-Lipschitz embeddable to $\mathbb{R}^{N}$. Bull. London Math. Soc. 34 (2002), no. 6, 667-676.

[20] Lang, U. And Plaut, C.: Bilipschitz embeddings of metric spaces into space forms. Geom. Dedicata 87 (2001), no. 1-3, 285-307.

[21] Lee, J.R., Mendel, M. And Naor, A.: Metric structures in $L_{1}$ : dimension, snowflakes, and average distortion. European J. Combin. 26 (2005), no. 8, 1180-1190.

[22] LeE, J. R. ANd NAOR, A.: $L_{p}$ metrics on the Heisenberg group and the GoemansLinial conjecture. In 47th Annual IEEE Symposium on Foundations of Computer Science (Berkeley, California, 2006), 99-108. Available at http://www.cims.nyu.edu/ naor/homepage\%20files/L_pHGL.pdf .

[23] Linial, N., London, E. and Rabinovich, Y.: The geometry of graphs and some of its algorithmic applications. Combinatorica 15 (1995), no. 2, 215-245.

[24] Naor, A. And Silberman, L.: Poincaré inequalities, embeddings, and wild groups. Compos. Math. 147 (2011), no. 5, 1546-1572.

[25] Pansu, P.: Métriques de Carnot-Carathéodory et quasiisométries des espaces symétriques de rang un. Ann. of Math. (2) 129 (1989), no. 1, 1-60.

[26] Schoenberg, I. J.: Metric spaces and positive definite functions. Trans. Amer. Math. Soc. 44 (1938), no. 3, 522-536.

[27] Semmes, S.: On the nonexistence of bi-Lipschitz parameterizations and geometric problems about $A_{\infty}$-weights. Rev. Mat. Iberoamericana 12 (1996), no. 2, 337-410.

[28] Semmes, S.: Bilipschitz embeddings of metric spaces into Euclidean spaces. Publ. Mat. 43 (1999), no. 2, 571-653.

[29] Talagrand, M.: Approximating a helix in finitely many dimensions. Ann. Inst. H. Poincaré Probab. Statist. 28 (1992), no. 3, 355-363.

Received December 16, 2010.

Assaf NaOR: Courant Institute, New York University, 251 Mercer Street, New York, NY 10012, USA.

E-mail: naor@cims.nyu.edu

Ofer Neiman: Computer Science Department, Ben-Gurion University, P.O. box 653, Be'er Sheva 84105, Israel.

E-mail: neimano@cs.bgu.ac.il 